\title{
A Mössbauer Effect Study of the Soledade Meteorite
}

\author{
C. Paduani, \\ Departamento de Física, Universidade Federal de Santa Catarina, UFSC, \\ Florianópolis, CEP 88040-900, SC, Brazil \\ C. A. Samudio Pérez, \\ Instituto de Ciências Exatas, UPF, Passo Fundo, CEP 99001-970, RS, Brazil
}

and J. D. Ardisson

Centro de Desenvolvimento da Tecnologia Nuclear, CDTN, Belo Horizonte, CEP 30123-970, MG, Brazil

Received on 24 May, 2005

\begin{abstract}
We performed a Mössbauer spectroscopy study of the iron meteorite Soledade. This meteorite, which consists of a metallic matrix, is an octahedrite with polycrystalline troilite, cohenite, schreibersite and rhabdites as major constituents. A chemical analysis indicates $6.78 \% \mathrm{Ni}, 0.46 \% \mathrm{Co}$, besides traces of $\mathrm{Cu}, \mathrm{Cr}, \mathrm{Ga}, \mathrm{Ge}, \mathrm{As}, \mathrm{Sb}$, $\mathrm{W}, \mathrm{Re}, \mathrm{Ir}$ and Au. No traces of silicates have been found and no oxygen was detected. Iron is appearing in the austhenitic phase and alloyed with nickel. An analysis of the Mössbauer spectra at room temperature indicates that the Fe-Ni phase is homogeneously distributed in the matrix, although variations in the composition between different regions are observed.
\end{abstract}

Since the early studies of the microstructure and chemical composition of meteorites the formation of magnetic phases has attracted the attention of metallurgists [1-11]. Most of the metallic specimens presented high contents of nickel and iron as major constituents, and thus the Fe-Ni alloys formed under such special conditions have been the subject of several investigations with a variety of experimental techniques. This is not an easy task considering the weathering process and the distribution of oxides in the metallic matrix, which in some cases varies in composition from one region to another. However, the complexity of the mechanism of formation of the alloys in meteorites, which can take cooling rates as long as $1 \mathrm{~K} / \mathrm{Ma}$, is an interesting subject, and its comprehension may shed some light on the metastability of alloys.

The category of iron meteorites corresponds to about $5 \%$ of the modern meteorite falls. In the study of Fe-Ni-bearing meteorites, there is a recent discussion about the formation of a low-moment Fe-rich $\gamma$ phase which differs from the ordinary high-spin $\gamma$ phase in the electronic structure and a lower lattice parameter, but has the same crystal structure, same degree of atomic order and same composition of ordinary taenite. In fact, this has been claimed to be a new mineral called antitaenite which is common in slowly cooled meteorites [12-14]. This low-spin phase is proposed to occur in a thin epitaxial intergrowth with tetrataenite (ferromagnetic atomically ordered FeNi). Actually, metastable precipitates of this low-spin phase in a matrix of high-spin $\mathrm{Fe}-\mathrm{Ni}$ phase of the same controlled composition have been synthetically produced near the Invar composition $(\approx 35$ at $\% \mathrm{Ni})$.

In this work we applied X-ray diffraction (XRD) and Mössbauer spectroscopy (MS) to study the iron-bearing phases detected in the iron meteorite called Soledade, which is a massive metallic block [15]. Although no one knows precisely when this specimen was found, it received the name of the locality from where it proceeded near the city of Passo Fundo in the state of Rio Grande do Sul in Brazil. The first studies indicate that this metallic meteorite is an octahedrite, with polycrystalline troilite, cohenite, schreibersite and rhab- dites as major constituents. It consists of a solid block weighing $68 \mathrm{~kg}$, with an irregular form measuring about $36 \times 22 \times 16$ $\mathrm{cm}$. A chemical analysis indicates $6.78 \% \mathrm{Ni}, 0.46 \% \mathrm{Co}$, besides traces of $\mathrm{Cu}, \mathrm{Cr}, \mathrm{Ga}, \mathrm{Ge}, \mathrm{As}, \mathrm{Sb}, \mathrm{W}, \mathrm{Re}, \mathrm{Ir}$ and $\mathrm{Au}$. No traces of silicates have been found. Iron is appearing in bcc phase alloyed with nickel. As pointed out, it has no relation with other meteorites which have been found in Brazil. It seems to have it has been suffered no violent impacts as well as no further re-heating was identified. To our knowledge, this is the first report on the Mössbauer spectroscopy in this specimen, which is intended to identify the Fe phases as well as to quantify these phases on the basis of their Mössbauer parameters.

In order to verify the differences in the iron-bearing phases which are present in the surface of the Soledade, three samples were taken from the outer portion of the meteorite, but interior to any surviving fusion crust. They are named thereafter as S1, S2 and S3. S1 was taken from one side ("top") of the specimen and S2 was taken from the opposite side ("bottom"), taking care to eliminate the coating of oxide which covers the metallic block. S3 is a sample collected from the coating of oxide. This has a reddish appearance, typical of iron oxides. A small amount of the fine powder was then sandwiched between adhesive tags in a lead holder, which was used as absorber for the MS measurements (width $\approx 50 \mu \mathrm{m}$ ). The spectra were obtained on a constant-acceleration spectrometer, using a $57-\mathrm{Co} / \mathrm{Rh}$ source, at $300 \mathrm{~K}$ (room temperature, RT) and $77 \mathrm{~K}$, being that 512 channels were used in the acquisition with a velocity increment of $0.068 \mathrm{~mm} / \mathrm{s} /$ channel. Drive velocity was calibrated at room temperature with a metallic $\alpha$ iron foil.

The XRD patterns were obtained with the $\mathrm{Cu}-\mathrm{K} \alpha$ radiation. The results for two samples (S2 and S3) are shown in Fig. 1. For the S1 and S2 samples these profiles are identical. The lattice parameters determined from these samples correspond to bec Fe-Ni alloys, which are similar to pure iron up to 10 at. $\% \mathrm{Ni}[16]$. Traces of iron oxide are detected for the S3 sample, 


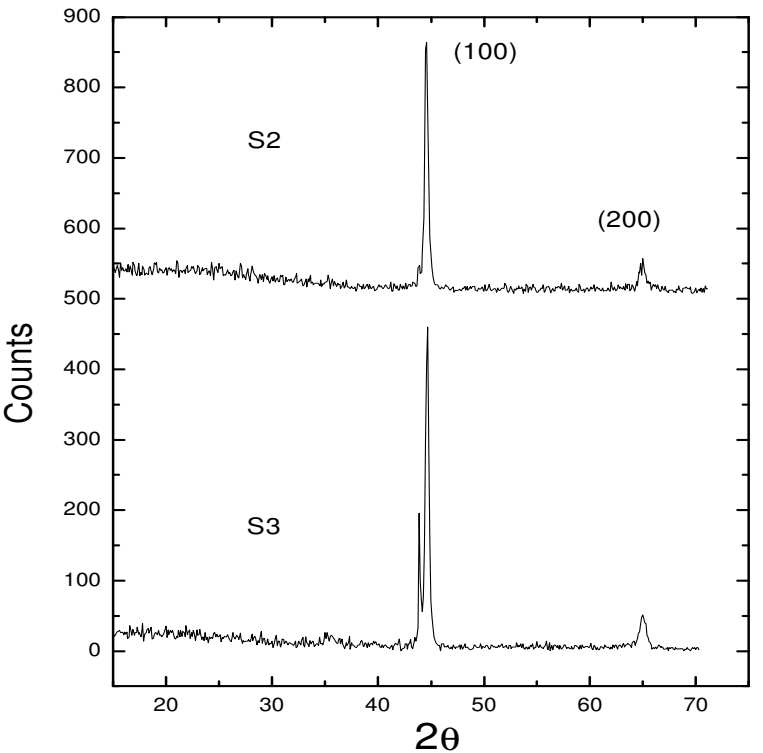

FIG. 1: XRD patterns for the Soledade meteorite. Top: S2 sample; bottom: S3 sample.

collected from the coating at the surface of the specimen.

The MS spectra registered at room temperature are shown in Fig. 2. The spectra were fitted with a constrained fitting program of Lorentzian functions which uses a nonlinear least-squares nonlinear minimization with the LevenbergMarquardt method (Normos package). A simple hyperfine field distribution was used to fit the spectra. This is a fast fit using only very simple asymmetry which is basically a histogram of sextets, with a possible linear change in isomer shift, where subspectra relative depths are calculated using linear regression plus damping. A block of 75 sextets with linewidths of $0.2 \mathrm{~mm} / \mathrm{s}$ was used to built up the overall spectrum. The first field value in the distribution block is $29 \mathrm{~T}$, which increases in steps of $0.1 \mathrm{~T}$. The others parameters are left free to adjust. In Fig. 2 are shown the fitted spectra together with the distribution profile at RT. The broad peak in the field distribution diagrams indicates that several contributions are adding to built up the overall envelope, which arises from different configurations of the iron sites in the specimen. The observed shift in this peak, when comparing the spectrum for S1 and S2, indicates that the magnetic phase is not homogeneously distributed in the block. However, the observed spectral shape for S1 and S2 are quite similar to that of a disordered $\mathrm{Fe}_{100-x} \mathrm{Ni}_{x}$ alloy for $\mathrm{x}<10$. The corresponding $\mathrm{H}_{h f}$ values also agree with those reported in the literature at this nickel concentration in $\mathrm{Fe}-\mathrm{Ni}$ alloys [16-17]. Therefore, for S3 one observes a change in the relative intensity of the inner lines of the sextets with respect to the outer lines, as well as the presence of a small paramagnetic component (doublet) at the center of the spectrum. The fitting procedure yields for it an isomer shift $\delta=0.14 \mathrm{~mm} / \mathrm{s}$ and a quadrupolar splitting $2 \varepsilon=$ $0.54 \mathrm{~mm} / \mathrm{s}$, with a spectral area $A=7 \%$. Actually, a tiny contribution from this paramagnetic phase can also be detected
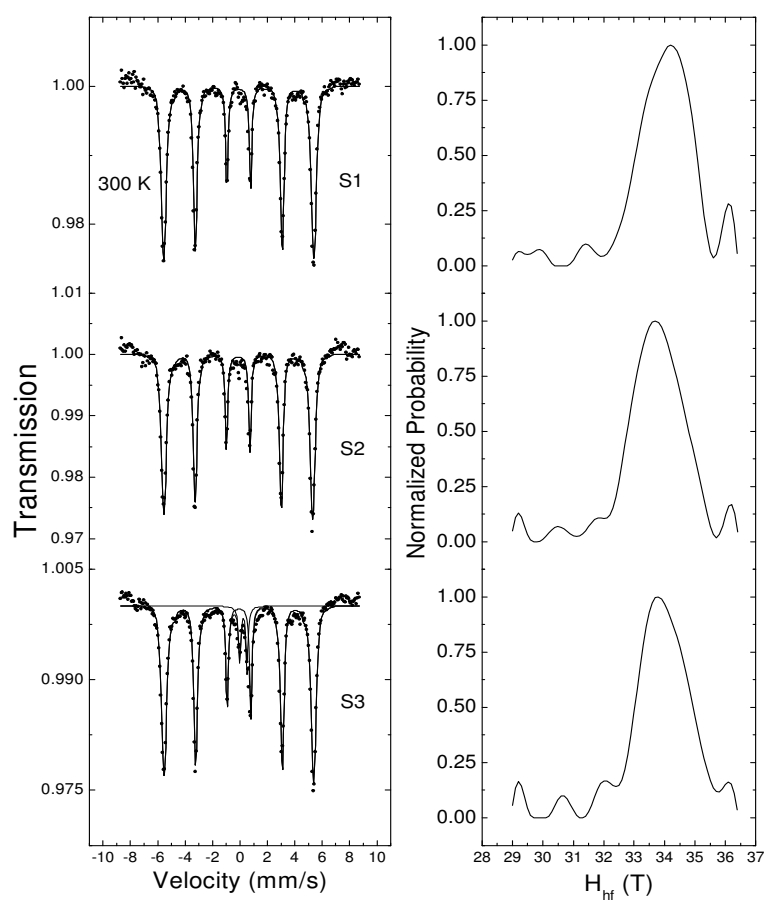

FIG. 2: Fitted MS spectra of the Soledade meteorite at $300 \mathrm{~K}$.

in the other samples. This can be ascribed to the presence of small particles of an iron oxide exhibiting a superparamagnetic behavior at RT. Besides, at $v \approx \pm 8.0 \mathrm{~mm} / \mathrm{s}$, small lines belonging to a sextet with a large field can also be identified, which is assigned to the presence of a magnetic iron oxide, probably the same as mentioned above, although with larger grain sizes.

The spectra taken at $77 \mathrm{~K}$ are shown in Fig. 3. The same fitting procedure used for the RT spectra was employed with the same line width. As can be seen in Fig. 3, the spectral shape for S2 and S3 is similar, and a small contribution from a paramagnetic phase still can be observed in the middle of the inner lines. However, at this temperature the spectral shape for the sample S3 has changed and becomes similar to that of the S1 and S2 samples. The central doublet has practically disappeared, and now is more explicit the presence of larger field values, in small proportion, which may be ascribed to the presence of iron oxides. The superparamagnetism exhibited by the small particles is suppressed at lower temperatures.

Hence, the metallic core of this octahedrite consists mainly of an iron-rich $\mathrm{Fe}-\mathrm{Ni}$ alloy, with magnetic hyperfine field values similar to those for as-cast alloys prepared by a conventional melting process. Despite the presence of traces of other metals, the formation of Fe-Ni alloys with continuous composition distribution was identified, whose Mössbauer parameters are similar to those observed in pure bcc $\mathrm{Fe}-\mathrm{Ni}$ alloys. Besides, the similarities of the Mössbauer spectra for the two samples collected in different parts of the metallic block also suggests that this specimen has a homogeneous distribution of Fe-Ni alloys in the volume, although small changes in 

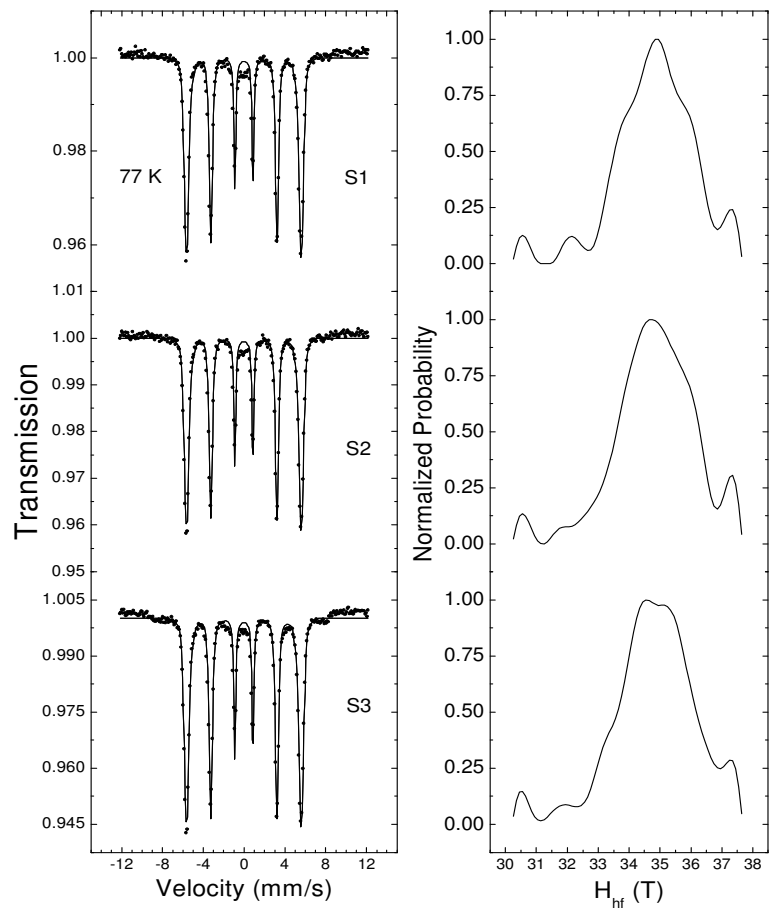

FIG. 3: Fitted MS spectra at $77 \mathrm{~K}$. the compositional distribution were observed. Thus, weathering has no significant effect on bulk elemental composition. In this case, there is no evidence of the presence of a lowmoment Fe-rich phase as it has been pointed out for the Santa Catharina meteorite (which is richer in iron). Finally, we would like to emphasize that a low-spin iron-rich phase was not detected in this study since our focus of attention was only in bulk samples (in natura). A chemical treatment of the samples is needed in order to eliminate the bcc phase and to obtain the lamelae (plate or needle-shaped crystals, formed during the slow cooling of meteoroids) containing the fcc phases[14] (tetrataenite and anti-taenite) which are expected to be present in all iron bearing meteorites. This can be the subject of a further investigation.

\section{Acknowledgement}

Partial financial support from the Brazilian agency CNPq is gratefully acknowledged. The authors would like to thank the Museu Muzar da UPF, RS, which gently provided the samples.
[1] J. F. Albertsen, G. B. Jensen, and J. M. Knudsen, Nature, 273 453 (1978)

[2] J. Danon, R. Scorzelli, and I. Souza Azevedo, An. Acad. Bras. Cien., 51, 176 (1979).

[3] J. Danon, R. Scorzelli, I. S. Azevedo, W. Curvello, J. F. Albertsen, and J. M. Knudsen, Nature, 277, 283 (1979).

[4] J. Danon, R.B. Scorzelli, I. Souza Azevedo, and K. Imakuma, Phys. Scripta 21, 223 ( 1980).

[5] R. B. Scorzelli, Thesis Docteur DÉtat, Université Pierre et Marie Curie, Paris 6, 1982.

[6] R. A. Jago, P. E. Clark, and P. L. Rossiter, Phys. Stat. Solidi (a) 74, 247 (1982).

[7] ] R. B. Scorzelli, J. Danon, Phys. Scripta 32, (1985)143.

[8] D. G. Rancourt, S. Chehab, and G. Lamarche, J. Magn. Magn. Mater. 78, 129 (1989).

[9] J. Zhang, D. B. Williams, J. I. Goldstein, and R. S. Clarke Jr, Meteoritics 25, 167 (1990).
[10] E. De Grave, R. E. Vandenburghe, P. M. A. de Bakker, A. Van Alboom, R. Vochten, and R. Van Tassel, Hyperfine Interactions 70, 1009 (1992).

[11] E. De Grave, R. J. Pollard, R. E. Vandenberghe, and P. M. A. de Bakker, Hyperfine Interactions 94, 2349 (1994).

[12] D. G. Rancourt, R. B. Scorzelli, J. Magn. Magn. Mater 150, 30 (1995).

[13] J. Chadwick, J. Magn. Magn. Mater. 174, 321 (1995).

[14] D.G. Rancourt, K. Lagarec, A. Densmore, R. A. Dunlap, J.I. Goldstein, R.J. Reisener, and R. B. Scorzelli, J. Magn. Magn. Mater 191, L255 (1999).

[15] M. E. Zucolotto, MsC. Thesis, UFRJ, 1988.

[16] J. Restrepo, G. A. Pérez-Alcazar, and A. Bohórquez, J. Appl. Phys. 81, 4101 (1997).

[17] I. Vincze, I. A. Campbell, and A. J. Meyer, Solid State Comm. 15, 1495 (1974). 